\title{
Evaluation of the efficacy of bite wafer chewing in pain reduction in fixed orthodontic appliance treatment
}

\author{
(1) Mukul Shetty ${ }^{1}$, (1) Sangamesh $\mathrm{B}^{2}$, (1) Akhil Shetty ${ }^{3}$, (1) Anand Patil ${ }^{4}$ \\ ${ }^{1} A B$ Shetty Memorial Institute of Dental Sciences, Clinic of Orthodontics and Dentofacial Orthopaedics, Deralakatte, India \\ 2AME's Dental College and Hospital, Clinic of Orthodontics and Dentofacial Orthopaedics, Raichur, India \\ ${ }^{3}$ Kanachur Hospital and Research Centre, Clinic of Dentistry, Deralakatte, India \\ 4SDM College of Dental Sciences and Hospital, Clinic of Orthodontics and Dentofacial Orthopaedics, Dharwad, India
}

Date submitted:

31.07.2019

Date accepted:

26.12.2019

Online publication date:

15.03.2020

\section{Corresponding Author:}

Mukul Shetty, AB Shetty Memorial Institute of Dental Sciences, Clinic of Orthodontics and Dentofacial Orthopaedics, Deralakatte, India mukulshetty28@gmail.com

ORCID:

orcid.org/0000-0003-3625-6916

Keywords: Bite wafers, gingival crevicular fluid, pain, periodontal ligament, substance $\mathrm{P}$

\begin{abstract}
Aim: The purpose of this study was to assess the efficacy of bite wafer (BW) in reducing pain levels, which is a highly complex and subjective phenomenon by assessing the substance $P$ level in the gingival crevicular fluid (GCF) at different time intervals after initial arch wire placement.

Methods: A parallel 2-group prospective case control study was designed for the estimation of substance $P$ levels in GCF after bite-wafer chewing to validate orthodontic pain reduction. The sample size consisted of 80 subjects (47 males and 33 females, mean age of $18.94 \pm 2.87$ years), who were randomly divided into two groups as the BW group (BWG) and the control group (CG). Fixed orthodontic appliance was placed in each patients of both groups and 0.014-inch nickel-titanium wire was placed and ligated. GCF was collected from the BW and CG before and 8, 24 and 72 hours after the initiation of orthodontic treatment. Unpaired T test was applied between the control and experimental group to evaluate the significant difference between the groups.
\end{abstract}

Results: The substance P level in GCF for both the BWG and CG followed a similar curve i.e., their levels increased after 8 hours, reached its peak at 24 hours and decreased gradually at 72 hours. The mean substance P level was significantly lower in the BWG compared to the CG, which implied that rhythmic chewing of BW helps in alleviating pain.

Conclusion: Bite wafers offer an excellent non-pharmacological option in reducing substance p level, thus indicating pain alleviation after orthodontic procedures.

\section{Introduction}

Pain and discomfort are often reported in patients undergoing fixed orthodontic treatment. Patients complain of pain interfering with their normal activities like biting, chewing, and sleep and some even consider terminating their treatment. Certain procedures like separator placement, placement and activation of arch wires are considered painful by the patients (1). Pain sensation usually begins by 2-4 hours following the placement of wire, and peaks at 24 hours; it gradually disappears over next 7 days (2). Pain perceived by the patient during orthodontic treatment is often ignored by the orthodontist and the option of pain-free treatment is not considered.
Studies have shown that pain following arch wire placement is the response due to the combination of pressure, edema, inflammation and ischemia in compressed periodontal ligament (PDL) (3). According to Proffit, pain experienced by the patients can be reduced by making them chew on the bite wafer (BW) repetitively for the first 8 hours after the activation of appliance (4). Chewing on the BW temporarily displaces the teeth thus allowing blood flow in the compressed areas of PDL, thus preventing build-up of pain-inducing metabolites.

Pain is a sensory experience that is highly subjective. The most commonly used method by a clinician to measure pain is visual analogue scale. Various factors such as age, sociocultural, psychological and environmental factors profoundly alter the 
perception of pain (5). Hence for accurate assessment of change in pain levels, substance $P$ is measured in gingival crevicular fluid (GCF). Substance $P$ is a neuropeptide which is found in GCF and is a inflammatory mediator (6). Substance $P$ plays an important role in the induction and maintenance of inflammation and dental pain (7). Studies have shown that levels of substance $P$ are elevated in painful teeth, indicating that they may have an important role in the mechanism of pain (8). Application of orthodontic forces significantly increases substance $P$ level in GCF after 8, 24 and 72 hours following orthodontic activation (9).

The use of non-steroidal anti-inflammatory drugs (NSAIDs) is the most preferred method for pain control in orthodontics but the overuse of analgesics and their potential side effects particularly in children have raised a concern over their use (10). Experimental and clinical studies have shown that NSAIDs can delay or inhibit tooth movement by inhibiting periodontal inflammatory response. Hence this clinical trial was conducted to evaluate the efficacy of BW in pain reduction by measuring substance $P$ level at different time intervals, i.e. before and 8, 24 and 72 hours after the placement of arch wire.

\section{Methods}

Patients reporting for the orthodontic treatment were included in this prospective study, after getting an approval from the Institutional Review Board (IRB) and Ethical Committee (IRB. no. 2012/P/OR/15) and patients' consent.

In this study 80 patients (47 males and 33 females, mean age of $18.94 \pm 2.87$ years) requiring fixed appliance therapy in at least one arch were consulted and selected. Only patients having mild to moderate crowding (1-6 mm of crowding) according to Little's irregularity index were included in this study (11). Informed consent was obtained from patients and parents/ guardians. Patients with periodontitis or any other periodontal problems were not included in the study. Patients with any skeletal anomalies were excluded from the study. Also patients on daily medication for systemic conditions or any medication taken 3-4 days before the start of the treatment or those undergoing any surgical procedures in the previous four weeks were excluded from the study.

The study was designed as a comparative prospective 2-group parallel clinical trial. The subjects were randomly divided into two groups of 40 subjects each. The first group was designated as the BW group (BWG) and the second as the control group (CG). The Bite-wafers used for study would be the standard Elastobyte wafers provided by the Ortho Technology, Inc. Florida, USA (Figure 1). All subjects received superficial prophylaxis three days before sample collection and they were instructed to follow good oral hygiene measures. All the subjects were bonded in at least one arch with Pre-Adjusted edgewise appliance with MBT prescription (0.022" slot) and 0.014" NiTi was used as the initial arch wire.
Subjects in the BWG were asked to bite or chew on the wafer for 8-10 minutes before the placement and then within an hour after the initial arch wire placement, and they were asked about experiencing any discomfort (Figure 2). They were specifically instructed to avoid any analgesic rescue medication. Substance $P$ levels in GCF was evaluated at 4 instances, i.e., pretreatment, 8 hours, 24 hours and 72 hours after the initial arch wire placement. The patients were made to sit comfortably on the dental chair with proper illumination.

The marginal gingiva was dried with a stream of air and isolated using cotton rolls. Average of $2 \mu \mathrm{L}$ of GCF was collected from the mesial as well as distal gingival crevices of the upper premolar using 1-5 $\mu \mathrm{L}$ calibrated volumetric micropipettes by capillary action (Figure 3 ). Each sample was examined upon removal and samples containing blood were discarded until an uncontaminated sample was obtained from each patient. The samples were diluted in phosphate buffer solution $(100 \mu \mathrm{L}, \mathrm{pH}$ :

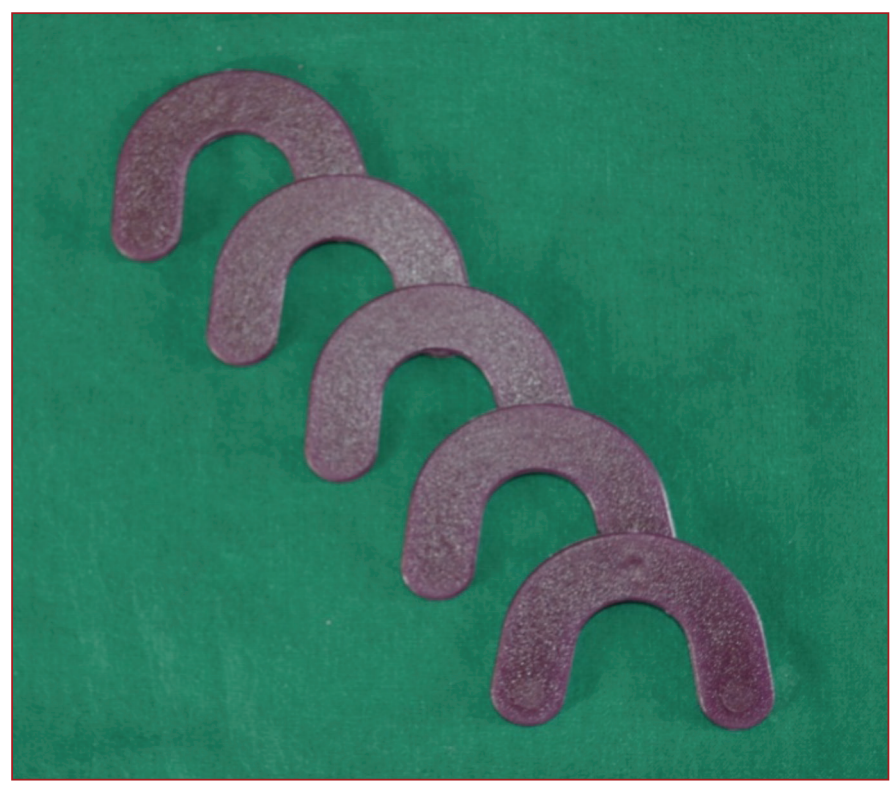

Figure 1. The Bite-wafers: The standard Elastobyte wafers by the Ortho Technology, Inc. Florida, USA

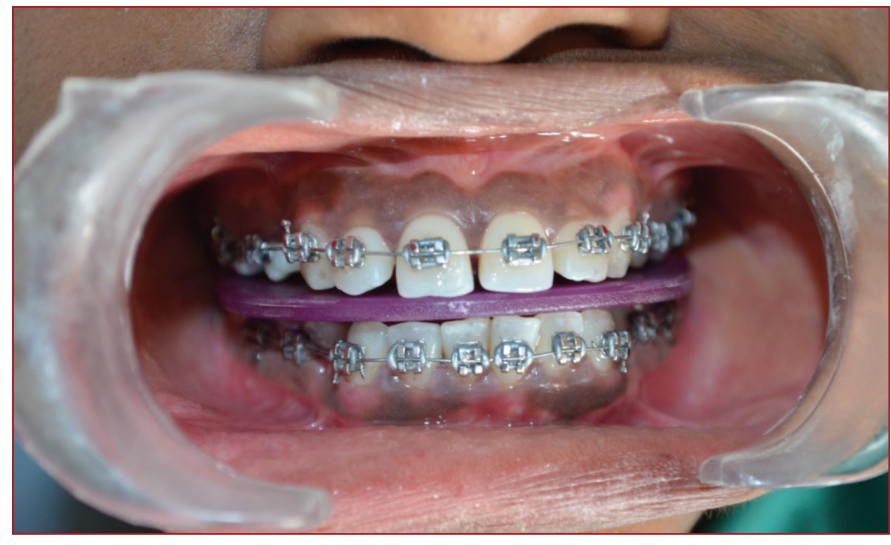

Figure 2. Patients in the Bite wafer group were asked to bite or chew on the wafer for 8-10 minutes 
7.2). The collected samples were stored at $-20{ }^{\circ} \mathrm{C}$ till analysis. Estimation of substance $P$ levels was done using ELISA (human substance $P$ immunoassay, R\&D Systems, Minneapolis, USA) according to manufacturer's instructions.

\section{Statistical Analysis}

All the data collected were transferred to a computer, the results were statistically analyzed using the statistical software SPSS (version 20.0 Armonk, NY: IBM Corp). The mean difference and standard error values of substance $P$ value were calculated at different time intervals. The comparison of the mean values between the two groups was performed using unpaired t test and within the groups at different time points ( 0 hours, 8 hours, 24 hours and 72 hours) by using ANOVA. A p value $<0.05$ was considered to indicate statistical significance.

\section{Results}

Results showed that the mean value of substance $P$ level in GCF reported by the BWG was less than the CG at 4 instances i.e., before the placement of arch wire, 8 hours, 24 hours and 72 hours after the initial arch wire placement. The reduced pain scores in the BWG could be attributed to the repetitive chewing of the wafer which caused temporary displacement of the teeth that allowed some blood flow through the compressed areas of the PDL, thus preventing the build-up of metabolites that could in turn stimulate pain receptors. Readings also showed that the mean value of substance $P$ level in GCF was maximum in both CG and BWG at 24 hours, indicating that maximum pain was perceived at 24 hours after the placement of arch wires (Graphic 1).

Unpaired t test showed statistically significant difference between the control and BWG at four different time intervals, i.e., T0, T1, T2, T3 since $\mathrm{p}<0.05$. This results indicate that subjects in the BWG showed substance $P$ level in GCF to be less than that of the $\mathrm{CG}$ at 4 different time intervals. Anova

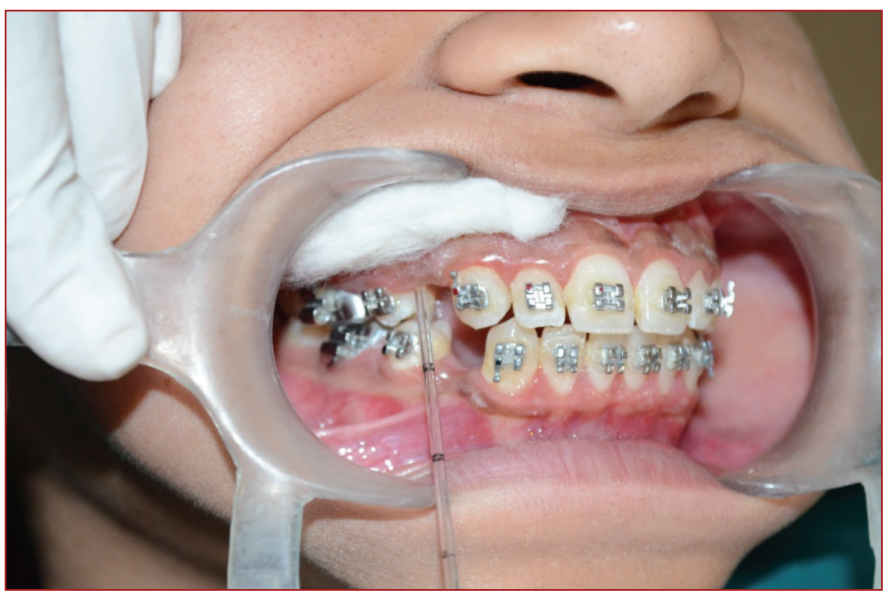

Figure 3. Gingival crevicular fluid was collected from the mesial as well as distal gingival crevices of the upper premolar using 1-5 $\mu \mathrm{L}$ calibrated volumetric micropipettes by capillary action test showed that substance $P$ level in GCF increased from the time orthodontic force was applied in both BW and CG groups, It reached its peak value at 24 hours and after that its value decreased gradually (Table 1 ).

Intragroup comparison of substance $P$ level in GCF samples among the CG at 4 different time interval showed that there was a significant difference between all pairs $(p=0.000,<0.05)$. This shows that the substance $P$ level was significantly higher at 8 hours, 24 hours and 72 hours. The values reached a peak at 24 hours after which they reduced, but still the value remained more than the baseline value (Table 2).

Intra group comparison of substance $P$ level in GCF samples among the BWG at 4 different time intervals showed that there was a significant difference between all pairs as $p<0.05$. This shows that the substance $P$ level was significantly higher at 8 hours, 24 hours and 72 hours. The values reached a peak at 24 hours after which the values dropped, but still the value remained more than the baseline value (Table 2 ).

\section{Discussion}

Pain is sensory experience that is very subjective. Fear of pain is highly common among patients undergoing any dental treatment. This fear of discomfort and pain discourage patients from undergoing orthodontic treatment (12). Discomfort and pain are experienced by some patients immediately after the placement of arch wire. Studies have shown that intensity of pain peaks at 24 hours following the application of force and it gradually decreases over the next seven days (13).

In orthodontics, the application of forces to induce tooth movement is the main cause of pain. The force applied to move the tooth through brackets and arch wire through alveolar bone may be perceived as a nociceptive input and might result in specific pain and inflammation receptors. On the application of orthodontic forces, ischemia, edema and inflammation occur in the compressed PDL, releasing mediators such as bradykinin

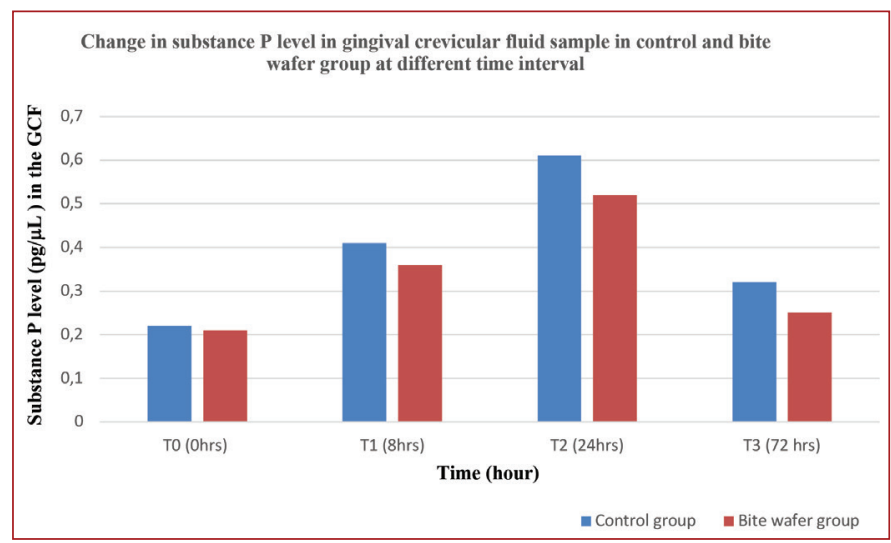

Graphic 1. Changes in substance $P$ level in gingival crevicular fluid in the control and bite wafer groups at different time intervals

GCF: Gingival crevicular fluid, hrs: Hours 


\begin{tabular}{|lllll|}
\hline \multicolumn{4}{|l|}{ Table 1. Unpaired test showing statistical difference in substance P level between the control and bite wafer groups } \\
\hline Group & T0 (0 hrs) & T1 (8 hrs) & T2 (24 hrs) & T3 (72 hrs) \\
\hline Control group & $0.22 \pm 0.02$ & $0.41 \pm 0.02$ & $0.61 \pm 0.02$ & $0.32 \pm 0.03$ \\
\hline Bite wafer group & $0.21 \pm 0.02$ & $0.36 \pm 0.02$ & $0.52 \pm 0.03$ & $0.25 \pm 0.02$ \\
\hline$t$ value & 1.31 & 9.27 & 12.70 & 9.56 \\
\hline$p$ value & 0.19 & $0.001^{*}$ & $0.001^{*}$ & $0.001^{*}$ \\
\hline $\begin{array}{l}* p<0.05 \text { is considered statistically significant. } \\
\text { hrs: Hours }\end{array}$ & & & \\
\hline
\end{tabular}

\begin{tabular}{|c|c|c|c|c|}
\hline Group & Time & Mean \pm SD & Wilk's Lambda & $p$ value \\
\hline \multirow[t]{4}{*}{ Control } & T0 (0 hrs) & $0.22 \pm 0.02$ & \multirow[t]{4}{*}{0.006} & \multirow[t]{4}{*}{$0.001^{*}$} \\
\hline & T1 (8 hrs) & $0.41 \pm 0.02$ & & \\
\hline & T2 (24 hrs) & $0.61 \pm 0.02$ & & \\
\hline & T3 (72 hrs) & $0.32 \pm 0.03$ & & \\
\hline \multirow[t]{4}{*}{ Bite wafer } & T0 (0 hrs) & $0.21 \pm 0.02$ & \multirow[t]{4}{*}{0.016} & \multirow[t]{4}{*}{$0.001^{*}$} \\
\hline & T1 (8 hrs) & $0.36 \pm 0.02$ & & \\
\hline & T2 (24 hrs) & $0.52 \pm 0.03$ & & \\
\hline & T3 (72 hrs) & $0.25 \pm 0.02$ & & \\
\hline
\end{tabular}

substance $\mathrm{P}$, prostaglandins, serotonin, histamine and this irritates the nerve ending of pain receptors which in turn causes pain (14).

Pain is a subjective response that shows a vast individual variations depending on the magnitude of force applied, individual pain threshold, gender, age, emotional state, cultural differences and previous pain experiences (15). The same stimuli may be perceived by different individuals as the amelioration or aggravation of pain. Thus it is difficult to measure pain which is a highly complex and personalized phenomenon.

Various methods have been recommended for controlling pain in orthodontic patients such as vibratory stimulation of PDL (16), low level laser therapy (17), NSAIDs (1), and transcutaneous electric nerve stimulation (18). Among these, NSAIDs were the most commonly used method for reducing pain, but due to side effects such as skin rash, headaches, thrombocytopenia, etc. seen with the use of these drugs especially in young patients, non-pharmacological approach like chewing on a BW is recommended for controlling orthodontic pain.

According to Stanfeld et al. (19), pain occurring during orthodontic treatment is due to inflammatory response taking place in PDL. In this study, we used BW to relieve pain because chewing on the BW reduced pain by causing loosening of the tightly grouped fibers around blood vessels and nerves, thus restoring normal vascular and lymphatic circulation, thus preventing and/or relieving inflammation and edema. According to Furstman and Bernik (3), orthodontic pain is a combination of pressure inflammation ischemia and edema. Chewing something hard can temporarily displace teeth, thus relieves the pressure and prevents the formation of ischemic areas which in turn releases pain.

An appreciable amount of substance $P$ is present in the GCF in teeth on which orthodontic force is applied (9). Substance $P$ level is increased in GCF during the incidence of pain (8). Studies have shown that initial orthodontic tooth movement incites pain and rapid release of biochemical mediators such as substance $\mathrm{P}$, interleukin 1B, prostaglandin $\mathrm{E} 2$, and substance $P$ in GCF. When orthodontic forces are applied, substance $P$ level is increased in GCF and its values were significantly higher after 8,24 , and 72 hours of orthodontic activation, with its peak value at 24 hours after which its value decreased by reaching almost its baseline measurement at 168 hours (9). Hence in this study, substance $P$ level in GCF was measured at 4 different time intervals, i.e. before the arch wire placement, 8 hours, after 24 hours, after 72 hours after the arch wire placement.

\section{Conclusion}

In the present study, it was found that there was a definite increase in substance $P$ level in GCF in both the CG and the BWG with its value increasing 8 hours after orthodontic force loading, its value peaked at 24 hours and then gradually decreased at 72 hours. Rhythmic chewing of BW reduced substance $P$ level in GCF in the BWG when compared to the CG at all the time intervals, thus indicating that BW chewing can be used to alleviate pain at any stage of orthodontic treatment and not just at the initial arch wire placement. The BW is an 
excellent non-pharmacologic option for pain management during orthodontic treatment without any of the side effects of analgesic medicines and it can easily be used at home or school without adult supervision.

\section{Ethics}

Ethics Committee Approval and Informed Consent: Patients reporting for the orthodontic treatment were included in this prospective study, after getting an approval from the Institutional Review Board (IRB) and Ethical Committee (IRB. no. 2012/P/OR/15) and patients' consent.

Peer-review: Externally peer-reviewed.

\section{Authorship Contributions}

Surgical and Medical Practices: S.B., Concept: M.S., Design: M.S., Data Collection or Processing: A.S., Analysis or Interpretation: A.S., Literature Search: A.P., Writing: S.B., M.S., A.S., A.P.

Conflict of Interest: No conflict of interest was declared by the authors.

Financial Disclosure: The authors declared that this study received no financial support.

\section{References}

1. Polat Ö. Pain and discomfort after orthodontic appointment. Seminar in Orthodontics. 2007;13:292-300.

2. Bergius $M$, Broberg $A G$, Hakeburg $M$, Berggren $U$. Prediction of prolonged pain experiences during orthodontic treatment. Am J Orthod Dentofacial Orthop. 2008;133:339.

3. Furstman L, Bernik S. Clinical considerations of the periodontium. Am J Orthod Dentofacial Orthop. 1972;61:138-155.

4. Proffit WR, Fields HW. Biologic basis of orthodontic therapy. Contemporary orthodontics. 5th ed. St Louis: Mosby; 2012.

5. Ngan P, Kess B, Wilson S. Perception of discomfort by patients undergoing orthodontic treatment. Am J Ortho Dentofacial Orthop. 1989;96:47-53.

6. Seybold VS. The role of peptides in central sensitization. Handb Exp Pharmacol. 2009:451-491.

7. Sacerdote P, Levrini L. Peripheral mechanism of dental pain: the role of substance P. Mediators Inflamm. 2012;2012:951920.

8. Rodd HD, Boissonade FM. Substance $P$ expression in human tooth pulp in relation to caries and pain experience. Eur J Oral Sci. 2000;108:467-474.
9. Yamaguchi M, Yoshii M, Kasai K. Relationship between substance $P$ and interleukin-1beta in gingival crevicular fluid during orthodontic tooth movement in adults. Eur $\mathrm{J}$ Orthod. 2006;28:241-246.

10. Raber A, Heras J, Costa J, Fortea J, Cobos A. Incidence of spontaneous notifications of adverse reactions with aceclofenac, meloxicam and rofecoxib during the first year after marketing in the United Kingdom. Ther Clin Risk Manag. 2007;3:225-230.

11. Little RM. The irregularity index: A quantitative score of mandibular anterior alignment. Am J Orthod. 1975;68:554563.

12. Brown DF, Moerenhout RG. The pain experience and psychological adjustment to orthodontic treatment of preadolescents, adolescents and adults. Am J Orthod Dentofacial Orthop. 1991;100:349-356.

13. Firestone AR, Scheurer PA, Burgin WB. Patients' anticipation of pain and pain related side effects, and their perception of pain as a result of orthodontic treatment with fixed appliances. Eur J Orthod. 1999;21:387-396.

14. Xiaoting L, Yin T, Yangxi C. Interventions for pain during fixed orthodontic appliance therapy, a systemic review. Angle Orthod. 2010;80:925-932.

15. Fernandes LM, Ogaard B, Skoglund L. Pain and discomfort experienced after placement of a conventional or a superelastic Niti aligning archwire. A randomized clinical trial. J Orofac Orthop. 1998;59:331-339.

16. Marie SS, Powers M, Sheridan JJ. Vibratory stimulation as a method of reducing pain after orthodontic appliance adjustment. J Clin Orthod. 2003;37:205-208.

17. Tortamano A, Lenzi DC, Haddad AC, Bottino MC, Dominguez GC, Vigorito JW. Low-level laser therapy for pain caused by placement of the first orthodontic archwire: a randomized clinical trial. Am J orthod Dentofacial Orthop. 2009;136:662-667.

18. Roth PM, Thrash WJ. Effect of transcutaneous electrical nerve stimulation for controlling pain associated with orthodontic tooth movement. Am J Orthod Dentofacial Orthop.1986;90:132-138.

19. Stanfeld J, Jones J, Laster L, Davidovitch Z. Biochemical aspects of orthodontic tooth movement: I. cyclic nucleotide and prostaglandin concentrations in tissues surrounding orthodontically treated teeth in vivo. Am $\mathrm{J}$ Orthod Dentofacial Orthop. 1986;90:139-148. 